\title{
Defining the focus of attention: effects of attention on perceived exertion and fatigue
}

\section{Keith R. Lohse* and David E. Sherwood}

Department of Psychology and Neuroscience, University of Colorado, Boulder, CO, USA

\section{Edited by:}

Jill Whitall, University of Maryland, USA

\section{Reviewed by:}

Nancy McNevin, University of

Windsor, Canada

Kevin Shockley, University of

Cincinnati, USA

${ }^{*}$ Correspondence:

Keith R. Lohse, Department of Psychology and Neuroscience, University of Colorado, Boulder, CO 80309, USA.

e-mail:keith.lohse@colorado.edu
This manuscript presents two experiments designed to explore the effects of attention on perceived exertion and time to failure in a fatiguing athletic task. There were two major motivating factors for these experiments. First, there are few studies evaluating attentional focus effects in endurance tasks and, second, there is a lack of integration between studies of attentional focus as external/internal (e.g., Wulf, 2007a) compared to associative/dissociative (e.g., Stevinson and Biddle, 1998). In Experiment 1, we used a fatiguing wall-sit posture (essentially a complex, isometric task) to compare two different types of external attention with an internal focus on the position of the legs. An external focus (regardless of type) increased the time taken to failure and reduced perceived exertion. In Experiment 2, we manipulated subjects' expectancy of fatigue to test the interaction of attention and expectancy (both top-down factors) in this highly fatiguing task. Previous theories of attention during endurance tasks have suggested that as fatigue/pain increase, bottom-up factors begin to dominate subjects' attention. While this may be true, Experiment 2 showed that even in a highly fatiguing task, attentional strategies, and expectancies affected the time to failure and perceived exertion.

Keywords: focus of attention, fatigue, expectancy

\section{DEFINING THE FOCUS OF ATTENTION: EFFECTS OF ATTENTION ON PERCEIVED EXERTION AND FATIGUE}

The focus of attention has been a burgeoning area of research in human movement science for the last decade. There is considerable evidence that directing attention externally to the effect of a movement on the environment (e.g., focusing on the flight of a ball in golf) improves performance compared to focusing internally on bodily movements involved in the execution of the motor skill (e.g., focusing on the motion of the arms in a golf swing). The advantage of an external focus of attention over an internal focus has been well documented across a wide variety of skills (for reviews see Wulf, 2007a; Lohse et al., 2012). Furthermore, a number of studies have shown an external focus of attention, induced through instructions and feedback by the experimenter, improves performance relative to uninstructed control conditions (McNevin and Wulf, 2002; Wulf and McNevin, 2003; Wulf et al., 2003; Landers et al., 2005), and the advantage of focusing externally holds true for recovering/performing motor skills in clinical populations, such as stroke (Fasoli et al., 2002) and Parkinson's disease patients (Landers et al., 2005; Wulf et al., 2009).

This research suggests that focusing externally is optimal for motor learning and skilled performance, although there is some debate about the optimal focus of attention for experts compared to novices. Research on how the focus of attention interacts with skill-level finds that experts can focus more externally, farther down the kinetic chain of events, than novices, who must focus more proximally (Wulf and Su, 2007; Bell and Hardy, 2009; or potentially internally; Perkins-Ceccato et al., 2003).

The exact mechanisms that underlie the effects of attention on skilled performance are not clear, but it has been suggested that focusing attention internally creates top-down constraints on the coordination of movement (known as the constrained action hypothesis; Wulf, 2007a,b). Research on muscle stiffness (Lohse et al., 2011b) and movement variability (Lohse et al., 2011a) suggest this might be at least partly true. However, we would contend that both an external focus and an internal focus place top-down constraints on the coordination of the motor system, the critical difference is in what movement dimensions are being constrained; is precision being controlled in bodily dimensions or orthogonal goal-dimensions that emerge from the interaction of the bodily dimensions (Lohse et al., 2011a)? This is a subtle, but significance change to the constrained action hypothesis.

Both of these theoretical positions, however, agree that there is more to focusing externally than simply distracting attention away from the body's movements. These theories posit that rather than simply being distracted away from movement, attention must be focused on some effect-relevant dimension of the task, based on action-effect representations in the motor system (e.g., commoncoding theory; Prinz, 1990). This position has been validated experimentally in studies that show subjects using a goal-relevant external focus of attention outperform subjects engaged in a distracting secondary task, who do no better than internally focused subjects (Wulf and McNevin, 2003; Castaneda and Gray, 2007). Thus, from the perspective of attention being directed internally or externally, distraction is not enough.

However, there is another, older body of research in human movement science dedicated to the focus of attention that studies attention in very different terms and focuses on distraction specifically. This research casts the problem of attention as a problem of what bodily/environmental cues a person should pay attention to 
in order to perform an arduous task successfully (Morgan, 1978; Weinberg et al., 1984). In this context of attention being directed to bodily/environmental cues, attention is described as either associative (focusing on bodily sensations involved in performance and more specifically, on physical sensations emanating from changes in temperature and muscular fatigue) or dissociative (a cognitive process of actively blocking out sensations of pain or discomfort related to physical effort; Weinberg et al., 1984).

Other researchers (Stevinson and Biddle, 1998, 1999) have added more detail to create a classification system for attention to bodily/environmental cues, by adding dimensions of relevance (task relevant or irrelevant) and direction of attention (either internal or external) which describes the locus of attention with respect to the body. Although the details of many of these models of attention are beyond the scope of this paper (see Leventhal, 1979; Leventhal and Everhart, 1979; Pennebaker and Lightner, 1980; Rejeski, 1985), in general these models suggest that there is competition between cues for access to awareness and attention can be directed either toward or away from certain cues (e.g., singing to myself while running rather than focusing on the burning in my quadriceps) by both top-down and bottom-up mechanisms (i.e., I can explicitly direct my cognition away from pain at sub-maximal thresholds, but as the exertion becomes more intense, bottom-up sensations play a larger role in determining the focus of attention; Tenenbaum, 2001; Ekkekakis, 2003).

Research on the optimal focus of attention (as associative or dissociative) has come to mixed conclusions. Some studies show higher ratings of perceived exertion (RPE) with an associative focus (Tammen, 1996; Schomer and Connolly, 2002; Baden et al., 2005). Other studies show higher RPE with a dissociative focus (Deligniéres and Brisswalter, 1994; Russell and Weeks, 1994; Brewer et al., 1996; Beaudoin et al., 1998), and still other studies show no difference (Weinberg et al., 1984; Harte and Eifert, 1995; Couture et al., 1999, 2003).

Other studies have focused on the effects of attention on physiological variables such as heart-rate and oxygen consumption. Again, the effects are mixed. Many studies have found no effect of attention on heart-rate or blood pressure (Pennebaker and Lightner, 1980; Weinberg et al., 1984; Johnson and Siegel, 1987; Hatfield et al., 1992; Baden et al., 2005). In contrast, Morgan et al. (1983) found that in the first 5-min of an incremental test on a treadmill, a dissociative focus led to lower heart-rate compared with a control condition. At the end of the test, however, this difference was eliminated. Similarly, some research shows that associative attention leads to increased heart-rate (Connolly and Janelle, 2003), but not in all instances (Couture et al., 1994). Studies that have looked at the effects of attention on oxygen consumption (e.g., $V_{\mathrm{O}_{2} \text { max }}$ generally find no effect of associative or dissociative attention on respiration (Morgan et al., 1983; Smith et al., 1995).

\section{PERSPECTIVES ON ATTENTION}

This brief review of the literature on the role of attention in motor learning and performance highlights an important problem: there are two largely separate branches of research studying attention in human performance that are (a) using identical language to describe different constructs and (b) do not integrate research from the other branch into their operational definitions, hypothesis, or theories. Consider when Wulf (2007b) uses the term external focus of attention to mean directing attention to the effect of an action on the environment contrasted with Stevinson and Biddle's (1998) use of the term to mean directing attention to an environmental cue outside the body. In Wulf's case it is attention directed toward efference (i.e., the intention to act) and in Stevinson and Biddle's case it is attention directed toward afference (i.e., the perception of cues).

Although these different conceptualizations may be divorced from each other in the laboratory, clearly they are never divorced from each other in practice. Indeed, focusing externally on the effect of an action on the environment may also weight the perception of external cues from the environment more heavily than internal cues from the body. In this way, focusing externally in the intention to act might also shift attention to external cues as corollary. In order to improve the understanding of how attention operates in complex behaviors, researchers need to integrate these different branches of human performance research. This was part of the motivation for the current experiments; we wanted to test these different conceptualizations of attention against each other using a fatiguing athletic task, focused on force production.

\section{EXPERIMENT 1}

Only a few experiments have studied the effects of attention on force production (for a review see Marchant, 2011). Based on evidence that an external focus of attention could reduce muscle activity in a basketball free-throw shot, while simultaneously increasing accuracy (Zachry et al., 2005), researchers speculated that similar external focus instructions might influence tests of maximal and sub-maximal force production. This hypothesis has been validated experimentally by a number of studies showing that sub-maximal forces in elbow flexion could be achieved with reduced surface electromyographic (sEMG) activity in the elbow flexor muscles (Vance et al., 2004; Marchant et al., 2009). A study of maximal force production in the form of a vertical jump also found that center-of-mass displacement was greater while sEMG activity in the musculature of the legs was reduced as a function of focusing externally (Wulf et al., 2010). Other studies have found that external focus of attention increases center-of-mass displacement in jumping, albeit without sEMG analysis (Wulf et al., 2007; Porter et al., 2010).

In our own laboratory, we have focused of studying accuracy in force production and found that focusing externally not only increases accuracy in the production of sub-maximal forces, but that an external focus of attention also leads to reduced cocontraction between agonist and antagonist muscles in plantar flexion (evaluated at 30\% maximum voluntary contraction, MVC; Lohse et al., 2011b), and that training with an external focus of attention significantly improves the retention and transfer of accuracy in force production (evaluated at 25 and 50\% MVC; Lohse, in press).

Limited data is available for tasks that manipulate the focus of attention in prolonged force production or endurance. Marchant et al. (2011) found that an external focus of attention (i.e., focusing on the motion of the bar vs. focusing on the motion of the body) significantly increased the number of repetitions to failure (with a load of $75 \%$ of maximum) in three different weight lifting 
exercises (presses on a Smith Machine bench press, a free weight bench press, and weighted squats). Schücker et al. (2009) found that an external focus of attention (i.e., focusing on a simulated environment) significantly improved running economy while running at an intensity of $75 \%$ of $V_{\mathrm{O}_{2} \text { max }}$ compared to an internal focus on form and an internal focus on breathing. This finding must be treated with some caution, however, because the simulated environment was only present during the external focus condition. Thus, the effect of an external focus was confounded with the presence of external cues.

In the current study, we aimed to address the relative dearth of literature evaluating attentional focus effects in endurance tasks, and the lack of integration between studies of attentional focus as external/internal compared to associative/dissociative. Thus, in the current study, we created a task to test muscular endurance (a wall-sit; shown in Figure 1) that could be completed under three different conditions: an internal focus (which is by nature associative), an external-associative focus, and an external-dissociative

\section{Experimental Foci: a. Internal Focus}

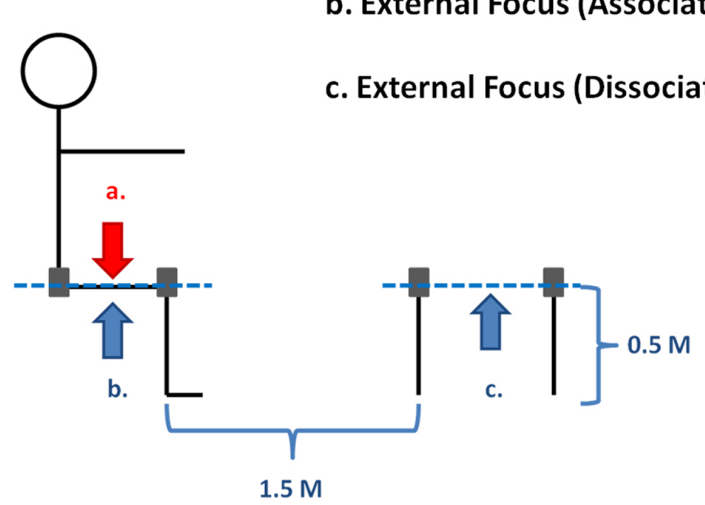

FIGURE 1 | A schematic representation of the experimental design, showing the three different attentional foci. The internal,

external-associative, and external-dissociative foci were all used in

Experiment 1. Only the internal and external-associative foci were used in Experiment 2. focus. These foci are illustrated in Figure 1 and explained in greater detail in the Section "Method." The wall-sit is a common exercise in athletics across a range of age and skill levels and creates a considerable amount of fatigue in the leg muscles very quickly. To measure performance in the wall-sit task, we measured the time that subjects could maintain the wall-sit position (referred to as time to failure) and subjects' self-reported RPE (assessed using a 15 point Borg scale; Borg, 1998).

\section{METHOD}

\section{Participants}

Data were collected from 40 healthy, physically active subjects at the University of Colorado. Of these, 21 were male and 19 were female. All subjects reported regularly engaging in exercise (three or more sessions per week) and six of the subjects played club sports at the university (varsity athletes were excluded from this study). The average age of the subjects was $19.25 \pm 1.28$ years (SD). (Demographic subject data are shown in Table 1.) Subjects were recruited through introductory psychology classes and participated in the experiment to fulfill course credit requirements. The study protocol was approved by the University of Colorado Institutional Review Board and all subjects gave informed consent prior to the experiment; subjects were naïve to the purposes of the experiment.

\section{Design}

After being given informed consent, the nature of the task was described to subjects. Subjects were told that they would do a "wall-sit, also known as an air-chair. You will stand with your back flat against the wall, walk your feet out until your knees and ankles are both at a $90^{\circ}$ angle and then hold this position for as long as you can." Most subjects reported being familiar with the task from previous athletic experience, but all subjects completed a short habituation trial (lasting $10 \mathrm{~s}$ ) prior to the start of the experimental trials. In the habituation trial, subjects first had reflective anatomical markers placed on the knee (at the lateral condyle of the femur) and the hip (greater trochanter of the femur) on each leg. Reflective markers were also placed on pylons $1.5 \mathrm{~m}$ in front of the subject (as shown in Figure 1).

Analog goniometers (Prestige Medical, Northridge, CA, USA) were used to make sure the knee and ankle angles were within

Table 1 | Summary of experimental and demographic data from Experiment 1.

\begin{tabular}{|c|c|c|c|c|}
\hline & \multicolumn{2}{|c|}{ Group $1(n=20)$} & \multicolumn{2}{|c|}{ Group $2(n=20)$} \\
\hline & External (assoc) & Internal & External (dissoc) & Internal \\
\hline Time (s) & $91.35 \pm 29.08$ & $84.98 \pm 26.87$ & $93.81 \pm 36.3$ & $86.54 \pm 30.61$ \\
\hline$R P E_{\text {initial }}$ & $8.25 \pm 1.94$ & $9.07 \pm 2.27$ & $8.92 \pm 1.86$ & $9.27 \pm 2.46$ \\
\hline$R P E_{\text {final }}$ & $17.55 \pm 1.61$ & $17.45 \pm 1.65$ & $16.90 \pm 2.13$ & $16.93 \pm 1.83$ \\
\hline Age (years) & \multicolumn{2}{|c|}{$19.20 \pm 1.61$} & \multicolumn{2}{|c|}{$19.05 \pm 0.88$} \\
\hline Height (cm) & \multicolumn{2}{|c|}{$173.41 \pm 6.86$} & \multicolumn{2}{|c|}{$169.42 \pm 11.80$} \\
\hline Weight (kg) & \multicolumn{2}{|c|}{$69.90 \pm 11.27$} & \multicolumn{2}{|c|}{$63.67 \pm 12.29$} \\
\hline
\end{tabular}

Table reports mean values $\pm S D$. 
$90^{\circ} \pm 5^{\circ}$ at the start of the trial. While holding this posture, subjects were given the following instructions, "Visually focus on the fixation point on the opposite wall [the experimenter gestured toward the opposite wall], while trying to keep your back flat against the wall. Also, you may not rest your hands on your legs, your hips, or against the wall behind you." Subjects were allowed to select any other position for their arms that felt the most comfortable, with most subjects simply letting their arms hang at their sides, but with some crossing their arms over their chest. Subjects could freely choose the position of their arms, but had to maintain the same position for all trials.

After completing the habituation trial, all subjects received the following instructions, "Today we will ask you to do wall-sits under two different conditions. You will only do two trials, and you will have a long rest period in between each one. The reason for a limited number of trials and a long rest period is that we want you to try your best to hold the wall-sit posture for as long as possible. Do not try to pace yourself; try your best on each trial. It is completely up to you when you want to end a trial. When you feel like you cannot hold the posture any longer, say 'Done' and then stand up or sit all the way down and the trial will be over. However, we do want you to make the trial last as long as possible."

Following these instructions, subjects began the experimental trials. The focus of attention was manipulated within-subjects (all subjects did one internally focused trial and one externally focused trial in a counterbalanced order) and the type of external focus was manipulated between subjects (half of the subjects had an external-associative focus and half of the subjects had an external-dissociative focus).

Internal focus instructions. For the internal focus (completed by all subjects), subjects were reminded to visually focus on the fixation point on the opposite wall but, "to mentally focus on the position of your thighs, trying to keep them parallel to the floor to minimize any movement up and down." Once the trial began, subjects were given feedback on the time every $30 \mathrm{~s}$ and reminded of their focus by being told to, "focus on the position of your thighs, trying to keep them parallel to the floor."

External-associative focus instructions. For the externalassociative focus (completed by half of the subjects), subjects were reminded to visually focus on the fixation point on the opposite wall but, "to mentally focus on the drawing imaginary lines between the markers from your knee to your hip, trying to keep the lines parallel to the floor to minimize any movement up and down." Once the trial began, subjects were given feedback on the time every $30 \mathrm{~s}$ and reminded of their focus by being told to, "focus on the position of the lines, trying to visualize them parallel to the floor."

External-dissociative focus instructions. For the externaldissociative focus (completed by half of the subjects), subjects were reminded to visually focus on the fixation point on the opposite wall but, "to mentally focus on the drawing imaginary lines between the pylons in front of you, trying to keep the lines parallel to the floor to minimize any movement up and down." Once the trial began, subjects were given feedback on the time every $30 \mathrm{~s}$ and reminded of their focus by being told to, "focus on the position of the lines, trying to visualize them parallel to the floor."

For all trials, after the instructions were given, subjects gave verbal confirmation that they understood the instructions and had to paraphrase the instructions back to the experimenter to verify they understood what was being asked of them. All subjects verified that they understood the instructions and were able to paraphrase task instructions for both the internal and external focus conditions. Thus, although it is not clear precisely how subjects used the internal/external focus strategies, subjects understood what was being asked of them in each condition.

\section{Apparati and measurement}

After verifying that subjects understood the instructions, subjects began their first experimental trial. Subjects stood with their back flat against the wall and visually focused on the fixation point on the opposite wall. Subjects then walked their feet out away from the wall until their knees and ankles were in the $90^{\circ} \pm 5^{\circ}$ range and the experimenter started the trial by starting the hand timer (S141, Seiko, Tokyo, Japan) and saying, "Begin." Every 30 s, the experimenter would remind subjects of the experimental focus and update subjects on the time. The experimenter visually monitored the position of the subjects legs, within the $90^{\circ} \pm 5^{\circ}$ tolerance, and subjects gaze, staying visually focused on the fixation point, throughout the trial. Subjects would verbally stop the trial by saying, "Done" when they reached the point of voluntary exhaustion. The time from the start of the trial to the end of the trial was defined as time to failure (measured in seconds).

At the end of each trial, the experimenter verbally surveyed subjects about their RPE using a 15 point scale (Borg, 1998). Subjects gave subjective RPE scores for how difficult the wall-sit felt in the first few seconds of the trial $\left(\mathrm{RPE}_{\text {initial }}\right)$ and at the very end of the trial when they had to stop ( $\left.\mathrm{RPE}_{\mathrm{final}}\right)$. Between trials subjects were allowed to rest for a minimum of $5 \mathrm{~min}$ or four-times the length of the previous trial (whichever time resulted in a longer rest period). Subjects were told this was the minimum time they had to rest between trials, but could take more time if they did not feel fully recovered. No subjects elected to take additional rest.

\section{Analysis}

Two dependent variables (time to failure and RPE) were analyzed. Time to failure was analyzed in a $2 \times 2 \times 2$ mixed-factorial ANOVA, with a within-subject factor of Focus (internal vs. external) and between-subjects factors of Order (external then internal or internal then external) and External Focus Type (associative vs. dissociative). RPE was analyzed in a $2 \times 2 \times 2 \times 2$ mixed-factorial ANOVA, with within-subject factors of Focus and Time (RPE initial vs. $\left.\mathrm{RPE}_{\mathrm{final}}\right)$ and between subject factors of Order and External Focus Type. Trial number was not included as a factor, but is statistically equivalent to the Order $\times$ Focus interaction because the order of the internal and external focus trials was fully counterbalanced across all subjects. Only significant effects are reported in the Section "Results." All other effects were non-significant $(p>0.05)$.

\section{RESULTS}

For time to failure, there was a significant main effect of focus, $F(1,36)=4.94, p=0.03, \eta_{p}^{2}=0.12$, shown in Figure 2. Focusing 
externally led to significantly longer time to failure than focusing internally. Interestingly, this effect did not depend on the type of external focus adopted, because the interaction of focus and external focus type was not significant, $F(1,36)<1$. This suggests that an associative-external focus is equally effective as a dissociative focus in alleviating subject responses to fatigue. However, there was a significant interaction of focus, external focus type, and order, $F(1,36)=4.38, p=0.04, \eta_{p}^{2}=0.10$, shown in Figure 2 . This interaction showed that, there was general trend for subjects to fatigue more quickly on the second trial. For the external then internal order, both external focus groups fatigued more quickly on the second trial. For the internal then external order, only the external-dissociative focus led to more rapid fatigue on the second trial. Interestingly, for the external-associative group, having the external-associative focus on the second trial seems to have alleviated the effects of fatigue, as their second trial was longer, on average, than the first trial. Although the interaction was significant, post hoc testing did not find any individually significant paired comparisons, although the advantages of the dissociativeexternal focus in the external-internal order and the associativeexternal focus in internal-external order approached significance (by Tukey's HSD, $p=0.09, p=0.07$, respectively). This interaction suggests there may be an advantage to an external-associative focus

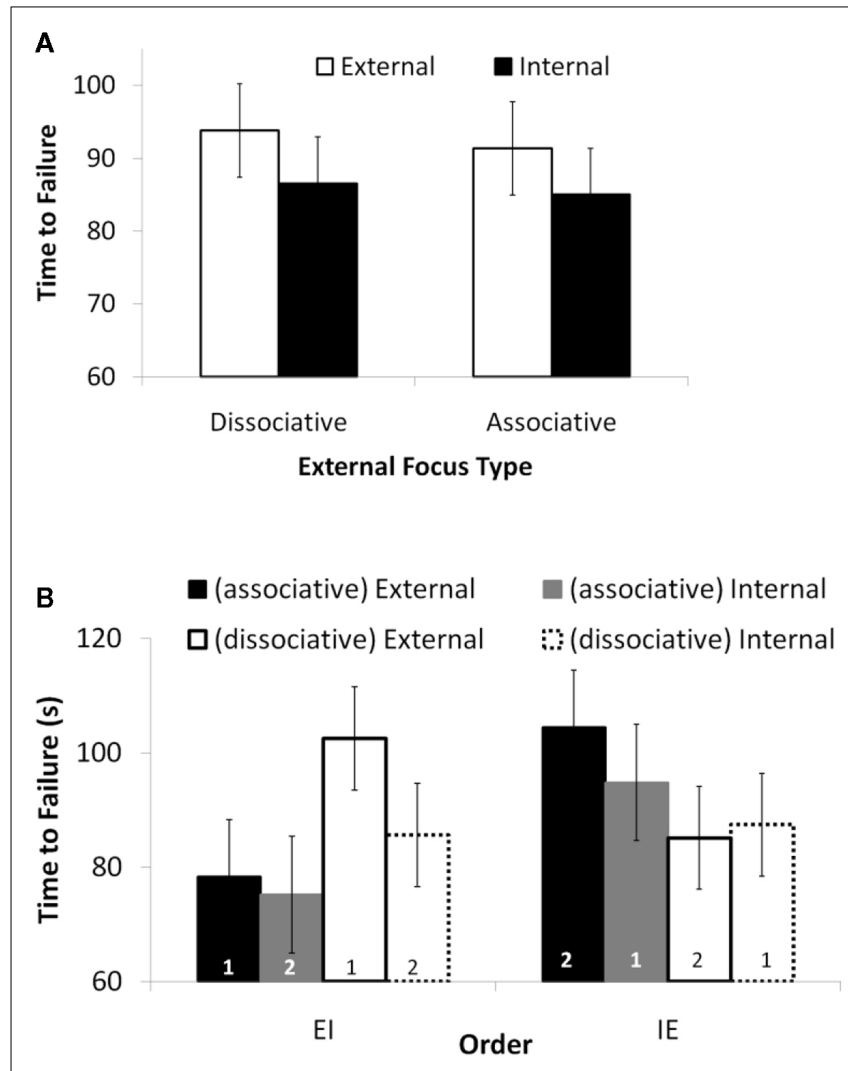

FIGURE 2 | Results of Experiment 1, showing time to failure as a function of attentional focus and externally focus type (A) and time to failure as a function of attention focus, external focus type, and trial (B). Trial numbers are shown at the bottom of each bar. Error bars show between-subjects SE. with increased fatigue. That is, with the external-associative focus, there is a larger advantage for focusing externally on the second trial.

Analysis of subjects' RPE showed a significant effect of time, $F(1,36)=716.91, p<0.001, \eta_{p}^{2}=0.95$, such that $\mathrm{RPE}_{\text {initial }}$ was significantly lower than $\mathrm{RPE}_{\mathrm{final}}$. There was a significant interaction of focus and order, $F(1,36)=44.69, p<0.001, \eta_{p}^{2}=0.55$, showing that the difference in average RPE between focusing internally and externally increased on the second trial (see Figure 3). Furthermore, there was a significant interaction of focus, order, and time, $F(1,36)=4.23, p=0.04, \eta_{p}^{2}=0.11$, such that on the first trial, if subjects were focusing externally their $\mathrm{RPE}_{\text {initial }}$ was lower than subjects focusing internally, but there was no difference in $\mathrm{RPE}_{\text {final }}$. On the second trial however, $\mathrm{RPE}_{\text {initial }}$ and $\mathrm{RPE} \mathrm{E}_{\text {final were }}$ both lower for externally focusing subjects (although individual paired comparisons showed that RPE was lower for the external focus compared to the internal focus in all cases, $p s<0.05$, see Figure 3). (Experimental and demographic data for Experiment 1 are summarized in Table $\mathbf{1}$ ).

\section{DISCUSSION}

Experiment 1 showed a clear benefit for focusing externally in both subjects' time to failure and subjects' perceived exertion during the task. Interestingly, subjects benefited from an external focus in both the associative and dissociative conditions. This result has important implications because it suggests that the primary benefit of focusing externally in this task was using distraction to block out internal sensations of fatigue which are made more salient by focusing internally (as shown in subjects' RPEs). Furthermore, this result is important because it expands on previous research on attention by showing that an external-associative focus, even though superficially very similar to the internal focus, increased time to failure and decreased RPE. This suggests that subjects can approximate an internal focus with a functionally similar but external focus. This is an important finding from an applied perspective because it suggests a mechanism by which coaches and athletes can

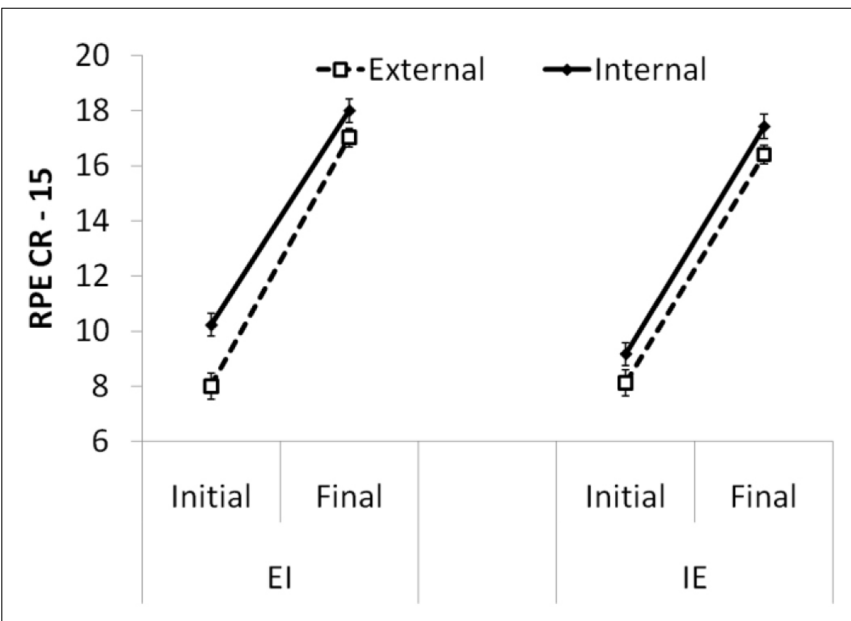

FIGURE 3 | Results of Experiment 1, RPE as a function of trial number, attentional focus and time (initial few seconds of the trial vs. final few seconds of the trial). 
reap the benefits of both focusing externally and focusing internally by using a nominally external focus that is analogous to an important internal variable. During rowing for instance, focusing on one's own body mechanics might increase the salience of internal cues and thus increase perceptions of fatigue, but focusing on an analog for one's mechanics (similar to the externalassociative focus) might allow for attention to be paid to control of the motion, while decreasing the salience of internal cues and decreasing perceptions of fatigue.

\section{EXPERIMENT 2}

The dual-mode model (DMM) of attention (Ekkekakis, 2003) suggests that at higher levels of fatigue, bottom-up processes direct attention more strongly than top-processes. That is, as pain or fatigue increase, internal, bodily signals of pain will dominate attention, even when an athlete is trying to maintain a dissociative, external focus on something in the environment. The DMM is supported by evidence that at low to moderate levels of exercise, there is a positive increase in the valence of basic affect, as well as a increase in the orthogonal dimension of activity (Raglin and Morgan, 1985; Ojanen, 1994), whereas at near-maximum levels of intensity, the shift in affective valence is uniformly negative (Acevedo et al., 1994; Parfitt et al., 1996). At moderate levels of exercise intensity, there are large inter-individual differences, with some subjects showing a positive shift in affect and other negative (Van Landuyt et al., 2000). These are the trends during exercise at different levels of intensity; post exercise, the data suggest a general positive shift in basic affect (Ekkekakis and Petruzzello, 1999).

Weighting bottom-up cues more heavily at more intense exercise intensities could play an adaptive function, because negative shifts in basic affect are correlated with increased metabolic demand on the body (Hardy and Rejeski, 1989; Acevedo et al., 1994; Ekkekakis and Petruzzello, 1999). Also, given the neural separability of affective systems in the brain (LeDoux, 1995, 2000), Ekkekakis (2003) suggests that two modes of processing interact with each other to determine affective responses to exercise: (a) a top-down, cognitive system that is involved in appraising the meaning of exercise, self-perceptions, goals, attributions, and the social context of exercise, and (b) a bottom-up, interoceptive system that feeds forward different cues from a variety of receptors based on exercise relevant physiological changes.

While there is certainly evidence that with increased intensity, internal cues become harder to block out of attention, in Experiment 2 the goal was to demonstrate that even at high level of exercise intensity there is still considerable top-down influence on performance. To test this, we chose to use the wall-sit task described in Experiment 1, because (looking at the RPE $\mathrm{final}_{\text {data) }}$ this task clearly creates a very intense demand on the body. In Experiment 1, we showed that a cognitive manipulation, directing attention either externally or internally through verbal instructions and feedback (attentional focus reminders given every $30 \mathrm{~s}$ ), improved or impaired performance respectively. In Experiment 2, our goal was to increase top-down influences on performance in this highly fatiguing task by manipulating subjects' expectations about the efficacy of their attentional strategy. That is, bias subjects to believe that either an external or internal strategy would be highly beneficial to their performance.
We hypothesized that biasing subjects to believe that an external focus is beneficial would substantially increase the benefit of focusing externally without bias. Further, we hypothesized that biasing subjects to believe that an internal focus is beneficial would reduce or even reverse the advantage normally found for focusing externally. These hypotheses were based on previous research showing the power of performance expectations using placebos in athletic tasks (Clark et al., 2000; Magnaris et al., 2000; Beedie et al., 2006; McClung and Collins, 2007; Foad et al., 2008). Most importantly, if bottom-up interoceptive information is the limiting factor on performance in this highly fatiguing task, we would expect no change in performance based on manipulating subjects expectations. Thus, if expectations augment or decrease the effect of attention, this would suggest a refining of the DMM, because top-down, cognitive factors would be influencing performance at very high levels of exertion.

\section{METHOD \\ Participants}

Sixty healthy, physically active subjects at the University of Colorado were recruited to participate in Experiment 2. Of these, 27 were male and 33 were female. All subjects reported regularly engaging in exercise (three or more sessions per week) and nine of the subjects played club sports at the university (varsity athletes were excluded from this study). The average age of the subjects was $19.15 \pm 1.31$ years. (Demographic subject data are shown in Table 2.) Subjects were recruited through introductory psychology classes and participated in the experiment to fulfill course credit requirements. The study protocol was approved by the University of Colorado Institutional Review Board and all subjects gave informed consent prior to the experiment; subjects were naïve to the purposes of the experiment.

\section{Design}

Subjects were divided into three groups, a control condition (in which subjects' expectations were unbiased), an external-good condition (in which subjects were biased to believe that focusing externally would be beneficial prior to the experimental trials), and an internal-good condition (in which subjects were biased to believe that focusing internally would be beneficial prior to the experimental trials). The experimental method was identical to that used in Experiment 1 for the external-associative group.

In order to induce biased expectations in the external-good and internal-good groups, subjects were shown data from a previous focus of attention experiment on isometric force production (Lohse et al., 2011b; recreated in Figure 4) and given the following explanation from the experimenter (in this case for the externalgood condition), "[...] These data show the results of a previous experiment on force production. The goal in this task was to produce a target force of $30 \%$ of that subject's maximum force, shown here [experimenter points to force-trace in figure] while recording muscle activity from the muscles of the lower leg [experimenter points to soleus and tibialis sEMG-traces in figure]. Subjects completed this task under two different conditions: an external focus, where subjects mentally focused on the platform they were pushing against, and an internal focus, where subjects mentally focused on their own leg. You can see that in the external focus [again, 
Table 2 | Summary of experimental and demographic data from Experiment 2.

\begin{tabular}{|c|c|c|c|c|c|c|}
\hline & \multicolumn{2}{|c|}{ Ctrl - no bias $(n=20)$} & \multicolumn{2}{|c|}{ Bias - external-good $(n=20)$} & \multicolumn{2}{|c|}{ Bias - internal-good $(n=20)$} \\
\hline & External & Internal & External & Internal & External & Internal \\
\hline Time (s) & $91.35 \pm 29.08$ & $84.98 \pm 26.87$ & $93.93 \pm 51.59$ & $80.59 \pm 45.46$ & $76.39 \pm 38.79$ & $84.16 \pm 48.98$ \\
\hline $\mathrm{RPE}_{\text {initial }}$ & $8.25 \pm 1.94$ & $9.07 \pm 2.27$ & $7.42 \pm 1.49$ & $8.07 \pm 2.01$ & $8.42 \pm 2.26$ & $8.33 \pm 2.28$ \\
\hline $\mathrm{RPE}_{\text {final }}$ & $17.55 \pm 1.61$ & $17.47 \pm 1.65$ & $16.27 \pm 1.91$ & $16.97 \pm 1.98$ & $16.07 \pm 1.83$ & $16.97 \pm 2.16$ \\
\hline Age & \multicolumn{2}{|c|}{$19.20 \pm 1.61$} & \multicolumn{2}{|c|}{$19.21 \pm 1.71$} & \multicolumn{2}{|c|}{$19.20 \pm 1.28$} \\
\hline Height & \multicolumn{2}{|c|}{$173.4 \pm 6.86$} & \multicolumn{2}{|c|}{$171.3 \pm 9.86$} & \multicolumn{2}{|c|}{$170.8 \pm 12.65$} \\
\hline Weight & \multicolumn{2}{|c|}{$69.90 \pm 11.27$} & \multicolumn{2}{|c|}{$67.2 \pm 12.67$} & \multicolumn{2}{|c|}{$67.41 \pm 11.94$} \\
\hline
\end{tabular}

Table reports mean values $\pm S D$.

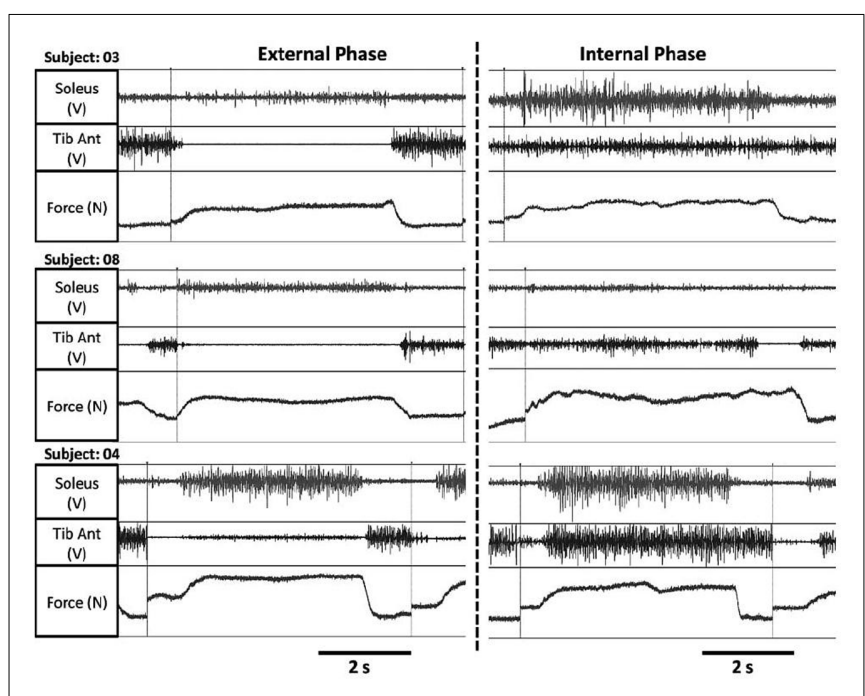

FIGURE 4 |A sample of materials used to bias subjects expectations in Experiment 2. This particular image is a recreation of the result from Lohse et al. (2011b) showing improved muscle recruitment efficiency with an external focus of attention (inducing an external-good bias). The same image was used to induce the internal-good bias, only the labels were reversed in the figure and the explanation given by the experimenter.

gesturing to the figure] the target force was produced with very little activity in the muscles of the leg. However, for the same subject trying to produce the same amount of force, the internal focus significantly increased muscle recruitment in the lower leg [gesturing to figure]. Thus, an external focus led to much more efficient muscle recruitment compared to an internal focus. In the experiment today, our goal will be to recreate this type of task using a wall-sit. You will do one external focus trial and one internal focus trial. We will begin with the [external/internal] focus trial [exact wording depended on counterbalancing order]." The internal-good bias condition received identical instructions only the labels "internal" and "external" were switched in both the verbal instructions and the figure shown to subjects.

After these biasing instructions, subjects were given the specific instructions for the internal and external focus trials. Internal focus instructions were identical to those used in Experiment 1, and the external focus instructions were identical to the externalassociative instructions in Experiment 1. Once the trial began, subjects were given feedback on the time every $30 \mathrm{~s}$ and reminded of their focus. For all trials, subjects gave verbal confirmation that they understood the instructions and had to paraphrase the instructions back to the experimenter to verify they understood what was being asked of them. A long rest period separated the first trial from the second trial (either 5 min or four-times the length of the first trial, whichever was longer). Again, this was the minimum time that subjects were required to rest, but could request more time if they were not fully recovered. No subjects elected to take additional rest.

\section{Analyses}

Time to failure and RPE data were collected in an identical method to Experiment 1. The external-associative group from Experiment 1 served as an unbiased control group for Experiment 2 . Thus, in the time to failure analysis there was a $2 \times 3 \times 2$ mixedfactorial ANOVA, with within-subject factors of Focus (external vs. internal) and between subjects factors of Bias (control, externalgood, or internal-good), and Order (either the external trial then the internal trial, or internal then external). For RPE, the was a $2 \times 2 \times 3 \times 2$ mixed-factorial ANOVA, with within-subject factors of Time ( $\mathrm{RPE}_{\text {intial }}$ vs. $\left.\mathrm{RPE}_{\text {final }}\right)$ and Focus, and between-subject factors of Bias and Order. Only significant effects are reported in the Section "Results." All other effect were non-significant $(p>0.05)$.

\section{RESULTS}

Analysis of subjects' time to failure revealed a significant interaction of focus and bias, $F(1,54)=7.13, p=0.002, \eta_{p}^{2}=0.21$, shown in Figure 5. This significant interaction revealed that the time to failure was $6.8 \mathrm{~s}$ longer for the external focus compared to the internal focus in the control condition, this difference was augmented in the external-good bias condition to $13.35 \mathrm{~s}$, and in the internal-good bias condition this relationship reversed, so that the time to failure was $7.76 \mathrm{~s}$ longer with an internal focus compared to an external focus. Post hoc Tukey's HSD testing revealed the difference between external and internal foci approached significance in the control condition ( $p=0.051)$, was highly significant for the external-good condition $(p=0.004)$, and was not significant for the internal-good condition $(p=0.081)$. 


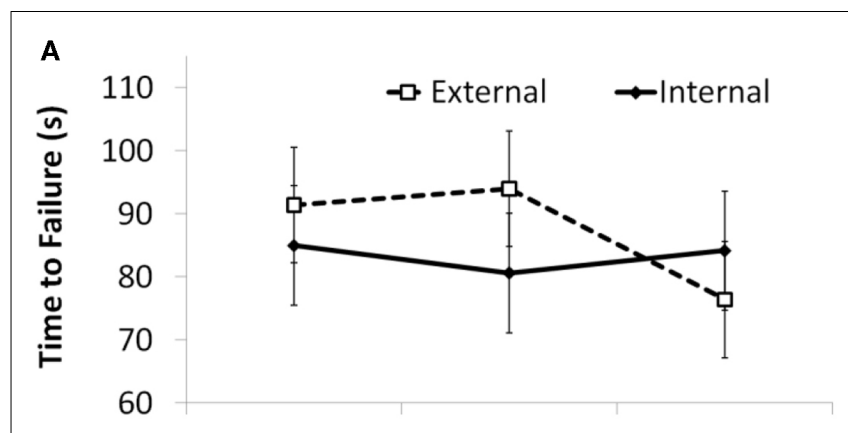

Control External Good Internal Good

Condition

B

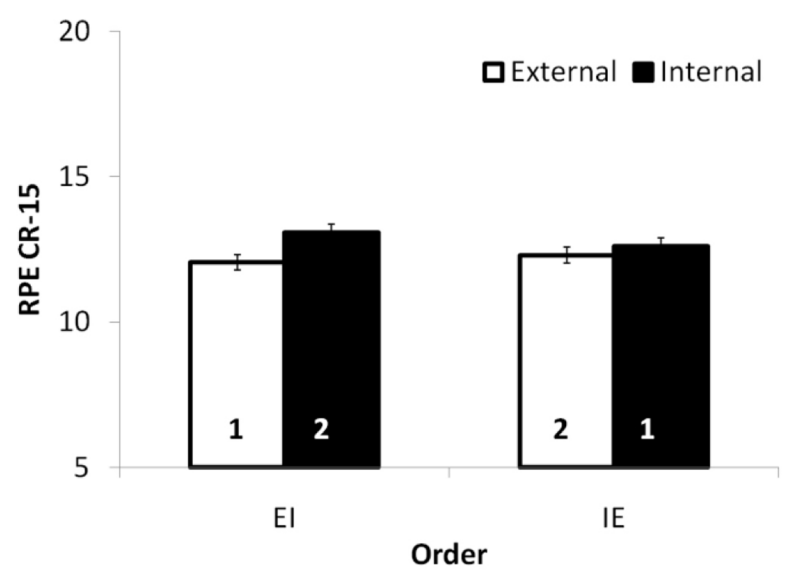

FIGURE 5 | Results of Experiment 2, showing time to failure as a function of attentional focus and bias condition $(A)$ and ratings of perceived exertion as a function of attentional focus and order (B). Trial numbers are shown at the bottom of each bar.

Analysis of subjects' RPE revealed a significant main effect of focus, $F(1,54)=4.22, p=0.038, \eta_{p}^{2}=0.08$, such that RPE ratings were lower for the external focus than the internal focus, on average. Focus significantly interacted with order, $F(1,54)=17.31$, $p<0.001, \eta_{p}^{2}=0.24$, such for subjects in the external-internal order, RPE for the external condition $(M=12.05)$ was significantly lower than the internal focus condition $(M=13.08 ; p<0.001)$. In the internal-external order, however, the two conditions were not significantly different from each other (External $=12.61$, Internal $=12.30 ; p=0.19$ ). See Figure 5. This is in contrast to Experiment 1 , in which the external-associative focus actually increased the difference between external and internal conditions when performed after the internal focus condition. This discrepancy needs to be addressed in future research, especially because the Focus $\times$ Order interaction in Experiment 2 averaged across three different groups, two of whom were biased by the experimental manipulation. Because no such manipulation took place in Experiment 1, it is difficult to directly compare the results of the two experiments.

Furthermore, there was a significant three-way interaction of focus, bias, and order, $F(2,54)=4.98, p=0.011, \eta_{p}^{2}=0.16$. Shown in Table 2, this interaction reveals that control subjects had lower $\mathrm{RPE}$ in the external focus condition in both orders, but this effect was only significant in the external-internal order $(p<0.001$ in the EI order; $p=0.06$ in the IE order). For external-good biased subjects, the external focus led to lower RPE only when the external focus trial was completed first $(p<0.004)$, when the internal focus trial was completed first, the difference in RPE all but disappeared $(p=0.61)$. For internal-good biased subjects, there was essentially no difference in RPE ratings when the external focus trial was completed first $(p=0.90)$, or when the internal focus trial was completed first $(p=0.54)$. Finally, there was also a highly significant effect of time, $F(1,54)=762.17, p<0.001, \eta_{p}^{2}=0.93$, such that $\mathrm{RPE}_{\text {intial }}$ ratings were much lower than $\mathrm{RPE}_{\text {final }}$ ratings. (This effect of time, and a summary of the experimental and demographic data from Experiment 2 are shown in Table 2.)

\section{DISCUSSION}

Experiment 2 showed a clear modulating effect of expectations on attentional focusing instructions. Although there was generally an advantage for focusing externally, as shown by the control group, this advantage can be augmented by biasing subjects' expectations about how effective an external focus of attention is. Conversely, this effect can also be wiped out by biasing subjects' expectations to believe that an internal focus is more advantageous. Importantly, attention affected not only subjective perceptions of exertion but also was shown to improve or impair objective measures of performance in subjects' time to failure.

The finding that manipulating subjects' expectations can have such profound effects on performance in a task with very high levels of exertion is not a contradiction of the DMM model of attention (Ekkekakis, 2003), but does suggest that the DMM needs to be refined, because top-down, cognitive factors clearly influence performance even at very intense levels of exertion (subjects' $\mathrm{RPE}_{\text {final }}$ averaged 16.88 when the maximum was 20 ). One issue that Experiment 2 did not address was how much of the advantage of external focus and expectation was due to efference and how much of the advantage was due to afference. To put it another way, from Experiments 1 and 2 we know that an external focus led to reduced perceptions of fatigue coincident to increased time to failure in a strenuous task. Thus, the mechanisms by which attention and expectancy were operating to improve performance are potentially perceptual. However, as the data from Lohse et al. (2011b) showed, attention can have significant effects of the efficiency of muscle recruitment, so it is possible that inefficient motor unit recruitment could have played a role in the current task as well. From these results, it is unknown if the decrease in RPE with an external focus represents any actual decreased muscular fatigue or if different attentional strategies changed only the subjective perception of fatigue. Because the current study made no physiological measurements of muscle fatigue, the current data cannot answer this question. However, this a very interesting area of future research because previous studies of expectations of performance in athletic tasks have not measured muscle activity (e.g., Magnaris et al., 2000; McClung and Collins, 2007; Foad et al., 2008). Thus, future research using expectancy manipulations and electromyography will be needed to disentangle the role of objective physiological fatigue and subjective perceptions of fatigue. 
These findings have applied significance for coaches, athletes, and therapists. Focusing externally generally improves a person's performance, but it is critical to address the individual's belief in the effects of attention. When subjects are focusing externally and they expect that focusing externally will facilitate performance (as it often does; Lohse et al., 2012) they do better than when focusing externally alone. The flip side of this effect is that even when focusing externally, if a subject does not believe that focusing externally is particularly useful (or in our case the subject believes that the internal focus is the most useful) the benefits of attention can be washed out by the effect of expectation.

This is one of few studies to address how the cognitive, affective, and motor systems interact (with manipulations of attention, expectations, and force production, respectively) and although it has led us to some exciting conclusions and new ideas about how these system work together in athletic performance, it is a study of a relatively limited task. Future research should work to address the integration of social, cognitive, affective, and motor systems in more diverse skills (continuous, discrete, ballistic, etc.) in order to understand the boundary conditions of these effects. Having a detailed taxonomy of how cognitive and affective variables affect performance in different domains of motor skills is important from a basic science perspective because it allows researchers to understand how these different systems influence behavior, but it is also supremely important from an applied perspective because in real-world motor behavior (e.g., athletics, rehabilitation, daily motor tasks) these systems are intricately and dynamically integrated.

\section{CONCLUSION}

This work confirms the efficacy of external focus of attention relative to an internal focus of attention, but adds new information

\section{REFERENCES}

Acevedo, E. O., Rhinehardt, K. F., and Kraemer, R. R. (1994). Perceived exertion and affect at varying intensities of running. Res. Q. Exerc. Sport 65, 372-376.

Baden, D. A., McLean, T. L., Tucker, R., Noakes, T. D., and St Clair Gibson, A. (2005). Effect of anticipation during unknown or unexpected exercise duration on rating of perceived exertion, affect, and physiological function. Br. J. Sports Med. 39, 742-746.

Beaudoin, C. M., Crews, D. J., and Morgan, D. W. (1998). Influence of psychogenic factors during a prolonged maximal run. J. Sport Behav. 21, 377-381.

Beedie, C. J., Stuart, E. M., Coleman, D. A., and Foad, A. J. (2006). Placebo effect of caffeine in cycling performance. Med. Sci. Sports Exerc. 38, 2159-2164.

Bell, J. J., and Hardy, J. (2009). Effects of attentional focus on skilled performance in golf. J. Appl. Sport Psychol. 21, 163-177.
Borg, G. (1998). Borg's Perceived Exertion and Pain Scales. Champaign, IL: Human Kinetics.

Brewer, B. W., Van Raalte, J. L., and Linder, D. E. (1996). Attentional focus and endurance performance. Appl. Res. Coach. Athl. Annu. 11, 1-14.

Castaneda, B., and Gray, R. (2007). Effects of focus of attention on baseball batting performance in players of differing skill levels. J. Sport Exerc. Psychol. 29, 60-77.

Clark, V. R., Hopkins, W. G., Hawley, J. A., and Burke, L. M. (2000). Placebo effect of carbohydrate feedings during a 40-km cycling time trial. Med. Sci. Sports Exerc. 32, 1642-1647.

Connolly, C. T., and Janelle, C. M. (2003). Attentional strategies in rowing: performance, perceived exertion, and gender considerations. J. Appl. Sport Psychol. 15, 195-212.

Couture, R. T., Jerome, W., and Tihanyi, J. (1999). Can associative and dissociative strategies affect the swimming performance of recreational swimmers? Sport Psychol. 13, 334-343.

to our understanding of attention in athletic performance and specifically during fatigue. Experiment 1 demonstrated focusing externally increased subjects' ability to resist fatigue, in part because an external focus reduced perceptions of fatigue, possibly as a result of distraction away from internal cues/sensations of fatigue. Furthermore, the use of an associative- and dissociativeexternal focus made no difference, and both external foci were significantly better than an internal focus. These results might change in a task with greater overt movement or when a specific environmental goal needs to be produced (e.g., Wulf, 2007b), whereas in the current task the goal was simply to resist fatigue for as long as possible. However, given the similarity between the external-associative focus and the internal focus, this finding suggests a useful way to reap the benefits of both an external focus and internal focus through the use of a conceptually external focus that is roughly analogous to an internal focus (the use of analogies has been shown to improve motor skill learning and performance in other domains; Liao and Masters, 2001; Poolton et al., 2006).

Experiment 2 showed that the relationship between FOA and performance can be modulated by expectations (dilating the effects of focusing externally or reducing them substantially). Both of these studies highlight the importance of top-down control in skilled motor performance and suggest that even though the salience of bottom-up cues may increase during stress, top-down factors continue to have significant influence in resisting fatigue. Future work is needed, however, that uses objective physiological measures of fatigue to disentangle potential effects that expectations and attention might have on motor unit recruitment in this task (similar to the effects of attention on motor unit recruit found in previous studies; Lohse et al., 2011b) from the effects that expectations and attention might have on the subjective perception of fatigue (as measured by RPE in the current experiments).

Couture, R. T., Singh, M., Lee, W., Chahal, P., Wankel, L., Oseen, M. and Wheeler, G. (1994). The effect of mental training on the performance of military endurance tasks in the Canadian infantry. Int. J. Sport Psychol. 25, 144-157.

Couture, R. T., Tihanyi, J., and St Aubin, M. (2003). Can performance in a distance swim be improved by increasing a preferred cognitive thinking strategy? Sport J. 6, 1-6.

Deligniéres, D., and Brisswalter, J. (1994). Influence of an added perceptual motor task on perceived exertion: a test of the dissociation effect. Percept. Mot. Skills 78, 855-858.

Ekkekakis, P. (2003). Pleasure and displeasure from the body: perspectives from exercise. Cogn. Emot. 17, 213-239.

Ekkekakis, P., and Petruzzello, S. J. (1999). Acute aerobic exercise and affect: current status, problems, and prospects regarding dose-response. Sports Med. 28, 337-374.
Fasoli, S. E., Trombly, C. A., TickleDegnen, L., and Verfaellie, M. H. (2002). Effect of instructions on functional reach in persons with and without cerebrovascular accident. Am. J. Occup. Ther. 56, 380-390.

Foad, A. J., Beedie, C. J., and Coleman, D. A. (2008). Pharmacological and psychological effects of caffeine ingestion in $40 \mathrm{~km}$ cycling performance. Med. Sci. Sports Exerc. 40, 158-165.

Hardy, C. J., and Rejeski, W. J. (1989). Not what, but how one feels: the measurement of affect during exercise. J. Sport Exerc. Psychol. 11, 304-317.

Harte, J. L., and Eifert, G. H. (1995). The effects of running, environment, and attentional focus on athletes' catecholamine and cortisol levels and mood. Psychophysiology 32, 49-54.

Hatfield, D. B., Spalding, T. W., Mahon, A. D., Slatter, B. A., Brody, E. B., and Vaccaro, P. (1992). The effect of psychological strategies upon cardiorespiratory and muscular activity during treadmill running. Med. Sci. Sports Exerc. 2, 218-225. 
Johnson, J. H., and Siegel, D. S. (1987). Active vs passive attentional manipulation and multidimensional perceptions of exercise intensity. Can. J. Sport Sci. 12, 41-45.

Landers, M., Wulf, G., Wallman, H., and Guadagnoli, M. A. (2005). An external focus of attention attenuates balance impairment in Parkinson's disease. Physiotherapy 91, 152-185.

LeDoux, J. E. (1995). Emotion: clues from the brain. Annu. Rev. Psychol. 46, 209-235.

LeDoux, J. E. (2000). Emotion circuits in the brain. Annu. Rev. Neurosci. 23, 155-184.

Leventhal, H. (1979). "A perceptualmotor processing model of emotion," in Perception of Emotion in Self and Others: Advances in the Study of Communication and Affect, eds P. Pliner, R. K. Blankstein, and I. M. Spigel (New York, NY: Plenum), $1-46$.

Leventhal, H., and Everhart, D. (1979). "Emotion pain, and physical illness," in Emotion and Psychopathology, ed. C. E. Izard (New York, NY: Plenum), 263-299.

Liao, C.-H., and Masters, R. S. W. (2001). Analogy learning: a means to implicit motor learning. J. Sports Sci. 19, 307-319.

Lohse, K. R. (in press). The influence of attention on learning and performance: pre-movement time and accuracy in an isometric force production task. Hum. Mov. Sci.

Lohse, K. R., Jones, M. C., Healy, A. F., and Sherwood, D. E. (2011a). Attention as a control parameter in the regulation of human movement. Paper presented at the North American Society for the Psychology of Sport and Physical Activity, Burlington.

Lohse, K. R., Sherwood, D. E., and Healy, A. F. (2011b). Neuromuscular effects of shifting the focus of attention in a simple force production task. J. Mot. Behav. 43, 174-184.

Lohse, K. R., Wulf, G., and Lewthwaite, R. (2012). "Attentional focus and movement efficiency," in Skill Acquisition in Sport: Research, Theory of Practice, 2nd Edn, eds N. J. Hodges and A. M. Williams (New York, NY: Routledge).

Magnaris, C. N., Collins, D., and Sharp, M. (2000). Expectancy effects and strength training: do steroids make a difference? Sport Psychologist 14, 272-278.

Marchant, D. C. (2011). Attentional focusing instructions and force production. Front. Psychol. 1:210. doi:10.3389/fpsyg.2010.00210
Marchant, D. C., Greig, M., Bullough, J., and Hitchen, D. (2011). Instructions to adopt and external focus enhance muscular endurance. Res. Q. Exerc. Sport 82, 466-473.

Marchant, D. C., Greig, M., and Scott, C. (2009). Attentional focusing instructions influence force production and muscular activity during isokinetic elbow flexions. J. Strength Cond. Res. 23, 2358-2366.

McClung, M., and Collins, D. (2007). "Because I know it will!": placebo effects of an ergogenic aid on athletic performance. J. Sport Exerc. Psychol. $29,382-394$.

McNevin, N. H., and Wulf, G. (2002). Attentional focus on supra-postural tasks affects postural control. Hum. Mov. Sci. 21, 187-202.

Morgan, W. P. (1978). Mind of the marathoner. Psychol. Today 11, 38-49.

Morgan, W. P., Horstman, D. H., Cymerman, A., and Stokes, J. (1983). Facilitation of physical performance by means of cognitive strategy. Cognit. Ther. Res. 7, 251-264.

Ojanen, M. (1994). Can the true effect of exercise on psychological variables be separated from placebo effects? Int. J. Sport Psychol. 25, 63-80.

Parfitt, G., Eston, R., and Connolly, D. (1996). Physiological affect at different ratings of perceived exertion in high-and low-active women: a study using a production protocol. Percept. Mot. Skills 82, 1035-1042.

Pennebaker, J. W., and Lightner, J. M. (1980). Competition of internal and external information in an exercise setting. J. Pers. Soc. Psychol. 39, 165-174.

Perkins-Ceccato, N., Passmore, S. R., and Lee, T. D. (2003). Effects of focus of attention depend on golfer's skills. J. Sports Sci. 21, 593-600.

Poolton, J. M., Masters, R. S. W., and Maxwell, J. P. (2006). The influence of analogy learning on decisionmaking in table tennis: evidence from behavioural data. Psychol. Sport Exerc. 7, 677-688.

Porter, J. M., Ostrowski, E. J., Nolan, R. P., and Wu, W. F. W. (2010). Standing long-jump performance is enhanced when using an external focus of attention. J. Strength Cond. Res. 24, 1746-1750.

Prinz, W. (1990). "A common coding approach to perception and action," in Relationships Between Perception and Action, eds O. Neumann and W. Prinz (Berlin: Springer-Verlag), 167-201.

Raglin, J. S., and Morgan, W. P. (1985). Influence of vigorous exercise on mood state. Behav. Ther. (N. Y. N. Y.) 8, 179-183.

Rejeski, W. J. (1985). Perceived exertion: an active or passive process? J. Sport Psychol. 7, 371-378.

Russell, W. D., and Weeks, D. L. (1994). Attentional style in ratings of perceived exertion during physical exercise. Percept. Mot. Skills 78, 779-783.

Schomer, H. H., and Connolly, M. J. (2002). Cognitive strategies used by marathoners in each quartile of a training run. S. Afr. J. Res. Sport Phys. Educ. Recreation 24, 87-99.

Schücker, L., Hagemann, N., Strauß, B. and Völker, K. (2009). The effect of attentional focus on running economy. J. Sport Sci. 27, 1241-1248.

Smith, A. L., Gill, D. L., Crews, D. J., Hopewell, R., and Morgan, D. W. (1995). Attentional strategy use by experienced long distance runners: physiological and psychological effects. Res. Q. Exerc. Sport 66, 142-150.

Stevinson, C. D., and Biddle, S. J. H. (1998). Cognitive orientations in marathon running and "hitting the wall.” Br. J. Sports Med. 32, 229-235.

Stevinson, C. D., and Biddle, S. J. H. (1999). Cognitive strategies in running: a response to Masters and Ogles (1998). Sport Psychol. 13, 235-236.

Tammen, V. V. (1996). Elite middle and long distance runner's associative/dissociative coping. J. Appl. Sport Psychol. 8, 1-8.

Tenenbaum, G. (2001). "A socialcognitive perspective of perceived exertion and exertion tolerance," in Handbook of Sport Psychology, eds R. N. Singer, H. A. Hausenblas, and C. Janelle (New York, NY: Wiley), 810-822.

Van Landuyt, L. M., Ekkekakis, P., Hall, E. E., and Petruzzello, S. J. (2000). Throwing the mountains into the lakes: on the perils of nomothetic conceptions of the exercise-affect relationship. J. Sport Exerc. Psychol. $22,208-234$.

Vance, J., Wulf, G., Töllner, T., McNevin, N., and Mercer, J. (2004). EMG activity as a function of the performer's focus of attention. J. Mot. Behav. 36, 450-459.

Weinberg, R. S., Smith, J., Jackson, A., and Gould, D. (1984). Effect of association, dissociation and positive self-talk strategies on endurance performance. Can. J. Appl. Sport Sci. 9, 25-32.

Wulf, G. (2007a). Attention and Motor Skill Learning. Champaign, IL: Human Kinetics.
Wulf, G. (2007b). Attentional focus and motor skill learning: a review of 10 years of research. Bewegung Training $1,4-14$.

Wulf, G., Dufek, J. S., Lozano, L., and Pettigrew, C. (2010). Increased jump height and reduced EMG activity with an external focus. Hum. Mov. Sci. 29, 440-448.

Wulf, G., Landers, M., Lewthwaite, R., and Töllner, T. (2009). External focus instructions reduce postural instability in individuals with Parkinson disease. Phys. Ther. 89, 162-168.

Wulf, G., and McNevin, N. H. (2003). Simply distracting learners is not enough: more evidence for the learning benefits of an external focus of attention. Eur. J. Sport Sci. 3, 1-13.

Wulf, G., and Su, J. (2007). External focus of attention enhances golf shot accuracy in beginners and experts. Res. Q. Exerc. Sport 78, 384-389.

Wulf, G., Weigelt, M., Poulter, D., and McNevin, N. H. (2003). Attentional focus on suprapostural tasks affects balance learning. Q. J. Exp. Psychol. 56, 1191-1211.

Wulf, G., Zachry, T., Granados, C., and Dufek, J. S. (2007). Increases in jump-and-reach height through an external focus of attention. Int. J. Sports Sci. Coach. 2, 275-284.

Zachry, T., Wulf, G, Mercer, J., and Bezodis, N. (2005). Increased movement accuracy and reduced EMG activity as a result of adopting an external focus of attention. Brain Res. Bull. 67, 304-309.

Conflict of Interest Statement: The authors declare that the research was conducted in the absence of any commercial or financial relationships that could be construed as a potential conflict of interest.

Received: 23 May 2011; accepted: 26 October 2011; published online: 14 November 2011.

Citation: Lohse KR and Sherwood DE (2011) Defining the focus of attention: effects of attention on perceived exertion and fatigue. Front. Psychology 2:332. doi: 10.3389/fpsyg.2011.00332

This article was submitted to Frontiers in Movement Science and Sport Psychology, a specialty of Frontiers in Psychology. Copyright $\odot 2011$ Lohse and Sherwood. This is an open-access article subject to a non-exclusive license between the authors and Frontiers Media SA, which permits use, distribution and reproduction in other forums, provided the original authors and source are credited and other Frontiers conditions are complied with. 